\title{
Placenta-specific 8 is a potential novel target for osimertinib resistance in non-small cell lung cancer
}

\author{
XIAOYUN FEI $^{1}$, GANG WANG $^{2}$, HUI SHEN $^{1}$ and XIAOHUA GU ${ }^{1}$ \\ ${ }^{1}$ Department of Respiratory Medicine, Shanghai Jiaotong University Affiliated Sixth People's Hospital; \\ ${ }^{2}$ State Key Laboratory of Bioreactor Engineering, School of Biotechnology, \\ East China University of Science and Technology, Shanghai 200233, P.R. China
}

Received February 8, 2018; Accepted April 25, 2019

DOI: $10.3892 / \mathrm{ol} .2019 .10344$

\begin{abstract}
Currently, osimertinib (AZD9291) is the only third-generation epidermal growth factor receptor (EGFR)-tyrosine kinase inhibitor approved by the Food and Drug Administration for the treatment of non-small cell lung cancer (NSCLC) with EGFR T790M mutations. However, acquired resistance is an inevitable clinical challenge. Although placenta-specific 8 (PLAC8) has been proven to serve an important role in tumor progression and resistance, its effect in AZD9291 resistance in NSCLC remains largely unknown. The aim of the present study was to investigate the functional role of PLAC8 in AZD9291 resistance in NSCLC. The results revealed that the level of PLAC8 was significantly upregulated in AZD9291-resistant cells compared with that in parent cells. Overexpression of PLAC8 in parent cells markedly decreased drug sensitivity, and enhanced cell proliferation, colony formation and migration. Furthermore, the levels of aldehyde dehydrogenase 1 family member A1 (ALDH1A1) were observed to be upregulated in resistant cells and PLAC8-overexpressing parent cells, suggesting that ALDH1A1 may be involved in the association between the overexpression of PLAC8 and AZD9291 resistance in NSCLC. Overall, PLAC8 overexpression promoted NSCLC resistance to AZD9291, and PLAC8 may be a potential target for the reversal of AZD9291 resistance.
\end{abstract}

\section{Introduction}

Lung cancer is the leading cause of cancer-associated mortality, and non-small cell lung cancer (NSCLC) accounts for $>80 \%$ of all lung cancer cases (1). Despite advances in chemotherapy and targeted therapy, the overall 5-year survival

Correspondence to: Dr Xiaohua Gu, Department of Respiratory Medicine, Shanghai Jiaotong University Affiliated Sixth People's Hospital, 600 Yishan Road, Shanghai 200233, P.R. China E-mail: gxhhzy@126.com

Key words: placenta-specific 8, osimertinib resistance, aldehyde dehydrogenase 1 family member A1, non-small cell lung cancer rate of lung cancer remains poor $(<15 \%)$, which is mainly attributed to resistance and metastasis $(2,3)$. Epidermal growth factor receptor (EGFR) mutations have been reported in $15 \%$ of NSCLC cases in Western countries, and in up to $40 \%$ of cases in Asian countries (4). Osimertinib (AZD9291) is a third-generation EGFR-tyrosine kinase inhibitor (TKI) that was approved by the Food and Drug Administration (FDA) in 2015 for the treatment of EGFR T790M-positive NSCLC patients (5). Inevitably, these patients eventually acquire resistance to AZD9291. Recent clinical data indicated that the emergence of C797S mutations has been identified in a subset of tresistant patients (6). However, other mechanisms contributing to the development of AZD9291 resistance in the majority of cases remain unknown.

Placenta-specific 8 (PLAC8) is a relatively small protein containing an evolutionarily-conserved cysteine-rich domain, which is expressed in human oocytes and preimplantation embryos, regulating placental and embryonic development $(7,8)$. Increasing evidence has demonstrated that PLAC8 is involved in the regulation of various cellular processes, and serves an important role in tumor progression and resistance (9-11). In colon cancer, elevated PLAC8 levels led to the development of epithelial-mesenchymal transition features and increased invasiveness, while knockdown of endogenous PLAC8 resulted in smaller tumors and reduced local invasion (12). Additionally, silencing of PLAC8 in renal cell carcinoma cells significantly increased their sensitivity to cisplatin (13). However, to the best of our knowledge, the functional role of PLAC8 in AZD9291 resistance in NSCLC has never been investigated.

The aldehyde dehydrogenase (ALDH) family is a group of enzymes that oxidize aldehyde into carboxylic acid and are involved in modulating early stem cell differentiation $(14,15)$. ALDH 1 family member A1 (ALDH1A1), a member of the ALDH family, has been demonstrated to be a marker for cancer stem cells (CSCs) in numerous types of tumors $(16,17)$. In lung CSCs, ALDH1A1 is upregulated and positively correlated with the stage and grade of lung cancer patients (18). Furthermore, ALDH1A1 was reported to be overexpressed in cisplatin-resistant lung cancer cell lines, and ALDH1A1 silencing significantly increased apoptosis and drug sensitivity (19). Taken together, these previous studies suggested that ALDH1A1 may contribute to AZD9291 resistance. 
In the present study, the expression levels of PLAC8 in AZD9291-resistant and AZD9291-sensitive NSCLC cell lines were analyzed. To the best of our knowledge, it was demonstrated for the first time that the PLAC8 levels were significantly upregulated in the resistant cells, indicating a potential regulatory role of PLAC8 in AZD9291 resistance in NSCLC. Subsequently, PLAC8 overexpression was induced in the AZD9291-sensitive NSCLC cells, and drug sensitivity and biological functions were assessed. Finally, the potential association between PLAC8 expression and AZD9291 resistance in NSCLC was preliminarily studied.

\section{Materials and methods}

Cell lines and culture. The AZD9291-resistant NSCLC cell lines PC9/AZD9291 and HCC827/AZD9291, as well as the respective sensitive cell lines PC9 and HCC827, were kindly provided by Dr Tianxiang Chen (Shanghai Chest Hospital, Shanghai, China). These four cell lines were cultured in RPMI-1640 medium (Invitrogen; Thermo Fisher Scientific, Inc., Waltham, MA, USA) supplemented with $10 \%$ fetal bovine serum (FBS; Invitrogen; Thermo Fisher Scientific, Inc.) and 1\% penicillin/streptomycin (Invitrogen; Thermo Fisher Scientific, Inc.). For resistance maintenance, $1 \mu \mathrm{mol} / 1 \mathrm{AZD} 9291$ (Selleck Chemicals, Houston, TX, USA) was also added to the culture of the two resistant cell lines. All cells were cultured at $37^{\circ} \mathrm{C}$ in a humidified atmosphere containing $5 \% \mathrm{CO}_{2}$.

Reverse transcription-quantitative polymerase chain reaction (RT-qPCR). RT-qPCR assay was performed to detect the relative mRNA expression levels of PLAC8 and ALDH1A1. Briefly, cells $\left(6 \times 10^{6}\right.$ cells/well) were incubated in a 6 -well plate for $24 \mathrm{~h}$, and then the total RNA was isolated using TRIzol reagent (Invitrogen; Thermo Fisher Scientific, Inc.), according to the manufacturer's protocol. The concentration of the total RNA was measured with a Nano-100 micro-spectrophotometer (Hangzhou Allsheng Instruments Co., Ltd., Hangzhou, China). Next, ReverTra ACE (Toyobo Life Science, Osaka, Japan) was applied to reverse transcribe the total mRNA into single-stranded cDNA. The qPCR assay was subsequently performed using a SYBR Premix Ex Taq ${ }^{\mathrm{TM}}$ II kit (Takara Biotechnology Co., Ltd., Dalian, China) in a final volume of $10 \mu \mathrm{l}$, containing $0.5 \mu \mathrm{l}$ cDNA, $0.5 \mu \mathrm{l}$ of each primer, $5 \mu \mathrm{l}$ SYBR Green and $3.5 \mu \mathrm{l}$ deionized water. The primers used were as follows: PLAC8 forward, 5'-GTGTGACTGTTTCAG CGACTG-3', and reverse, 5'-CTGCAACTTGACACCCAA GG-3'; ALDH1A1 forward, 5'-GCACGCCAGACTTACCTG TC-3', and reverse, 5'-CCTCCTCAGTTGCAGGATTAA AG-3'; GAPDH forward, 5'-GGAGCGAGATCCCTCCAA AAT-3', and reverse, 5'-GGCTGTTGTCATACTTCTCAT GG-3'. Relative mRNA expression was normalized to GAPDH expression, which served as the internal control. The reactions were performed in a 96 -well optical plate at $95^{\circ} \mathrm{C}$ for $5 \mathrm{~min}$, followed by 42 cycles of $95^{\circ} \mathrm{C}$ for $5 \mathrm{sec}$ and $60^{\circ} \mathrm{C}$ for $1 \mathrm{~min}$. Relative quantification of gene expression was performed by the $2^{-\Delta \Delta \mathrm{Cq}}$ method (20).

Western blot analysis. Western blot assay was performed to evaluate the relative protein expression levels of PLAC8 and ALDH1A1. Briefly, cells $\left(6 \times 10^{6}\right.$ cells/well) were incubated for $24 \mathrm{~h}$ in a 6 -well plate and the lysates were prepared with radioimmunoprecipitation assay buffer (containing $50 \mathrm{mM}$ Tris- $\mathrm{HCl}, \mathrm{pH} 8.0,150 \mathrm{mM}$ sodium chloride, 1.0\% IGEPAL CA-630, $0.5 \%$ sodium deoxycholate and $0.1 \%$ sodium dodecyl sulfate; Sigma-Aldrich; Merck KGaA, Darmstadt, Germany), followed by centrifugation $\left(13,800 \mathrm{x} \mathrm{g} ; 10 \mathrm{~min} ; 4^{\circ} \mathrm{C}\right)$. Total protein concentration was determined using a Bradford assay. Next, $20 \mu \mathrm{g}$ protein was separated by SDS-PAGE (15\% gel) and subsequently transferred to polyvinylidene fluoride membranes. The membranes were incubated with the corresponding primary antibodies at $4^{\circ} \mathrm{C}$ overnight, followed by incubation with horseradish peroxidase-conjugated secondary antibodies for $1 \mathrm{~h}$ at room temperature. The primary antibodies, including PLAC8 (cat. no. 13885; 1:1,000), ALDH1A1 (cat. no. 54135; 1:1,000) and GAPDH (cat. no. 5174; 1:1,000), and the secondary antibody (cat. no. 7074; 1:2,000) used in the assay were obtained from Cell Signaling Technology, Inc. (Danvers, MA, USA). GAPDH was used as a loading control to measure the expression of each protein. Bands were visualized using Pierce ${ }^{\mathrm{TM}}$ ECL Western Blotting Substrate (Pierce; Thermo Fisher Scientific, Inc.). Gray values of the blot bands were then analyzed using ImageJ software (National Institutes of Health, Bethesda, MD, USA), and the relative gray value was used to represent the relative protein expression.

Lentivirus production and infection. Full-length human PLAC8 was amplified and cloned into the PLVX-mCherry-N1 plasmid (Clontech Laboratories, Inc., Mountainview, CA, USA). 293T cells were subsequently transfected with lentiviruses containing PLAC8-PLVX and packaging plasmids using Lipofectamine ${ }^{\circledR} 2000$ (Invitrogen; Thermo Fisher Scientific, Inc.), following the manufacturer's protocols. The supernatant medium containing lentivirus was collected by centrifugation $\left(5,000 \mathrm{x} \mathrm{g} ; 60 \mathrm{~min} ; 4^{\circ} \mathrm{C}\right)$ to remove cellular contaminants. The resulting lentiviruses were used to infect the sensitive cell lines PC9 and HCC827, and then successfully infected cells were selected using $2 \mu \mathrm{g} / \mathrm{ml}$ puromycin (Sigma-Aldrich; Merck KGaA). Positive clones were screened and verified by RT-qPCR analysis. Cells overexpressing PLAC8 were termed PC9-PLAC8 and HCC827-PLAC8, while control cells were termed PC9-CTL and HCC827-CTL.

Cell proliferation. Cells were digested and seeded at a density of 3,000 cells/well in 96-well plates. Then cells were cultured for 1, 2, 3, 4, 5, 6 and 7 days, separately, and $10 \mu 1$ 3-(4,5-dimethylthiazol-2-yl)-2,5-diphenyltetrazolium bromide (MTT; Sigma-Aldrich; Merck KGaA) was added to the appropriate wells and incubated for another $3 \mathrm{~h}$. Subsequent to discarding the supernatant, $100 \mu 1$ dimethyl sulfoxide (DMSO; Sigma-Aldrich; Merck KGaA) was added to the cells, and the absorbance was detected at $492 \mathrm{~nm}$. Cell proliferation was evaluated using the following equation: Proliferation rate $=\mathrm{A}$ (sample) $/ \mathrm{A}$ (control).

Cell viability. Cells were seeded in 96-well plates $(5,000$ cells/well $)$ and cultured overnight. The transfected cells were treated with $0,0.01,0.05,0.1,0.5,1$ and $10 \mu \mathrm{M}$ AZD9291 respectively the following morning and incubated for $72 \mathrm{~h}$. Subsequently, $10 \mu 1$ MTT was added to each well and incubated for a further $3 \mathrm{~h}$. The supernatant was then removed and $100 \mu \mathrm{l}$ 

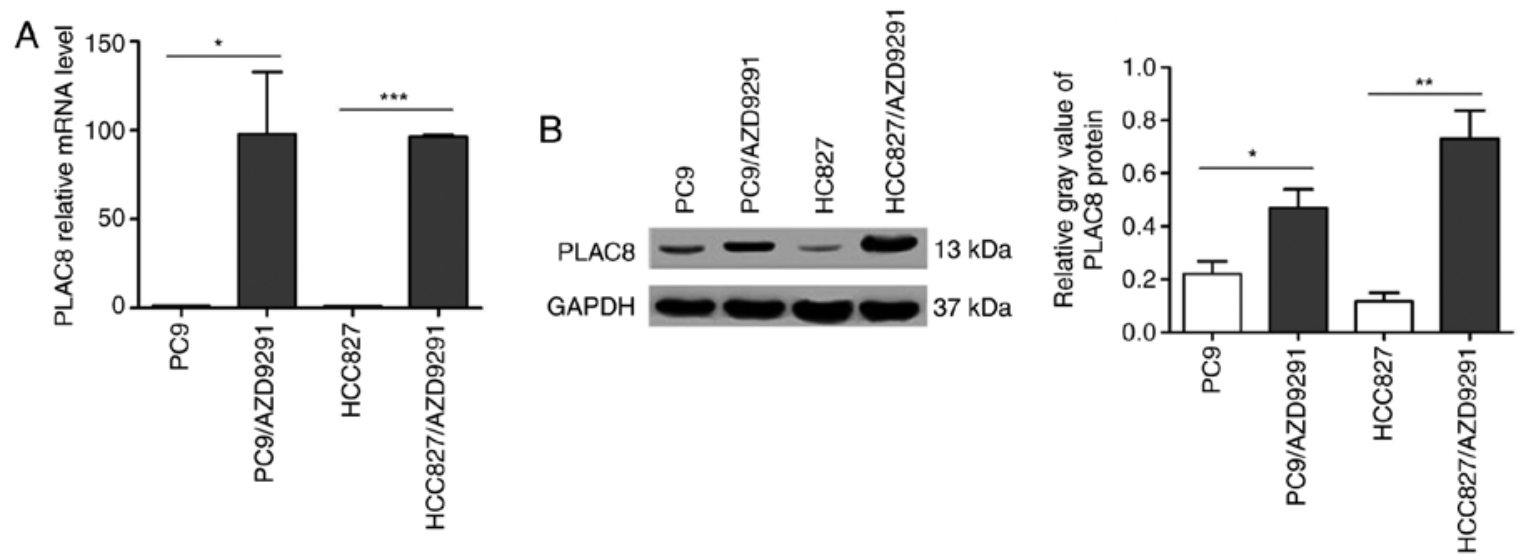

Figure 1. PLAC8 expression is upregulated in AZD9291-resistant NSCLC cell lines. (A) mRNA and (B) protein expression levels of PLAC8 in parent and AZD9291-resistant NSCLC cells. ${ }^{*} \mathrm{P}<0.05,{ }^{* *} \mathrm{P}<0.01$ and ${ }^{* * * *} \mathrm{P}<0.001$. PLAC8, placenta-specific 8; AZD9291, osimertinib; NSCLC, non-small cell lung cancer.
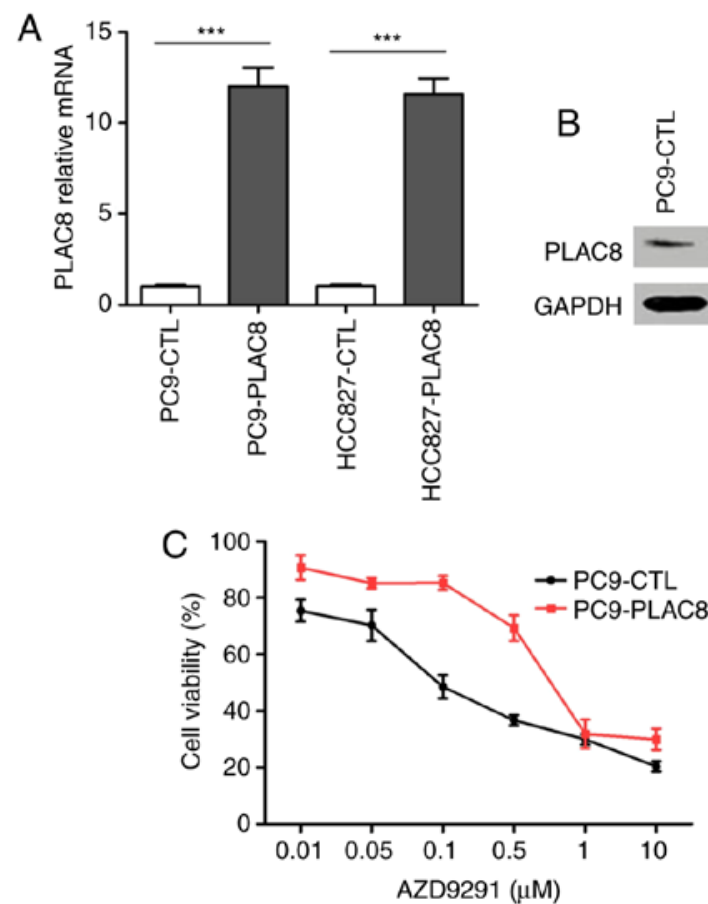
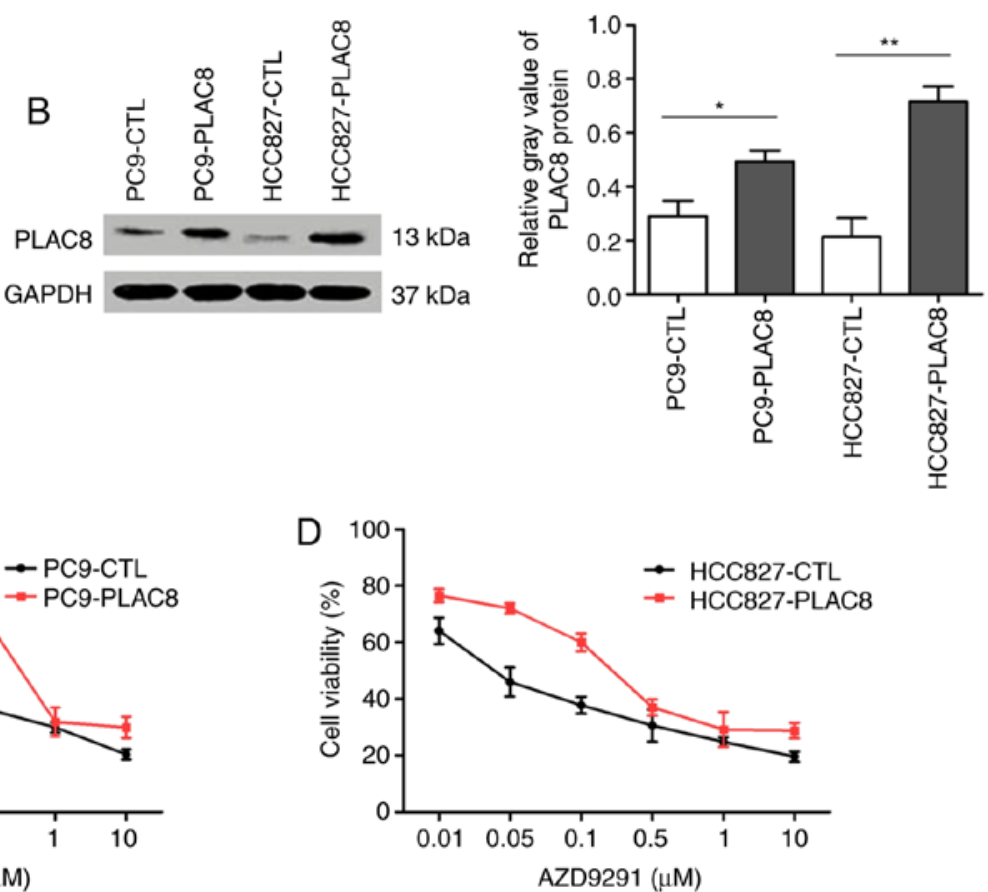

Figure 2. Overexpression of PLAC8 promotes AZD9291-resistance in NSCLC cells. (A) mRNA and (B) protein expression levels of PLAC8 in control and PLAC8-overexpressing NSCLC cells. (C) PC9 and (D) HCC827 viability in control and PLAC8-overexpressing cell lines. The cells were treated with different concentrations of AZD9291 for $72 \mathrm{~h}$, and then an MTT assay was applied to detect cell viability. ${ }^{*} \mathrm{P}<0.05,{ }^{* *} \mathrm{P}<0.01$ and ${ }^{* * *} \mathrm{P}<0.001$. PLAC8, placenta-specific 8; AZD9291, osimertinib; NSCLC, non-small cell lung cancer; CTL, control.

DMSO was added to each well. Finally, the absorbance was measured at $492 \mathrm{~nm}$, and the cell viability was determined as follows: Cell viability $(\%)=\mathrm{A}($ sample $) / \mathrm{A}($ control $) \times 100 \%$. The half-maximal inhibitory concentration $\left(\mathrm{IC}_{50}\right)$ was also calculated with SPSS software (version 16.0; SPSS, Inc., Chicago, IL, USA). Resistance index was then calculated as follows: Resistance index $=\mathrm{IC}_{50}$ (cells overexpressing $\left.\mathrm{PLAC} 8\right) / \mathrm{IC}_{50}$ (corresponding control cells).

Colony formation assay. Cells were seeded in 6-well plates (400 cells/well) and incubated for 10-14 days, until visible colonies were observed. The medium was replaced every 3 days during the culture period. Subsequently, the plates were washed with PBS, and the colonies were fixed with methanol for $20 \mathrm{~min}$, stained with $1 \%$ crystal violet for $30 \mathrm{~min}$ and counted. The number of colonies containing $>50$ cells was determined.

Migration assay. The migration assay was performed using 24-well transwell inserts $(8-\mu \mathrm{m}$ pore size; EMD Millipore, Billerica, MA, USA), according to manufacturer's protocol. Briefly, $2 \times 10^{4}$ cells/well suspended in serum-free RPMI-1640 medium were seeded in the upper chamber, and RPMI-1640 containing $10 \%$ FBS was added to the lower chamber. Following incubation for $24 \mathrm{~h}$, cells on the upper surface of the membrane were removed. Cells remaining on the bottom surface were fixed with methanol for $20 \mathrm{~min}$ and then stained with a $1 \%$ crystal violet solution for $0.5 \mathrm{~h}$. Finally, the stained 
A

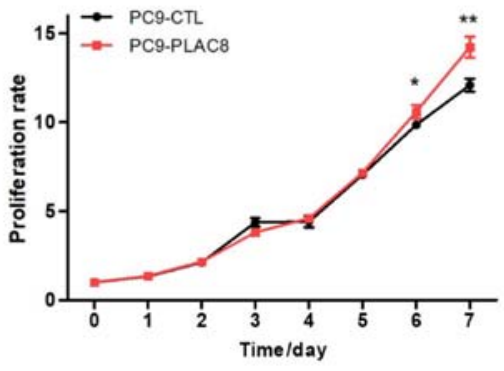

C

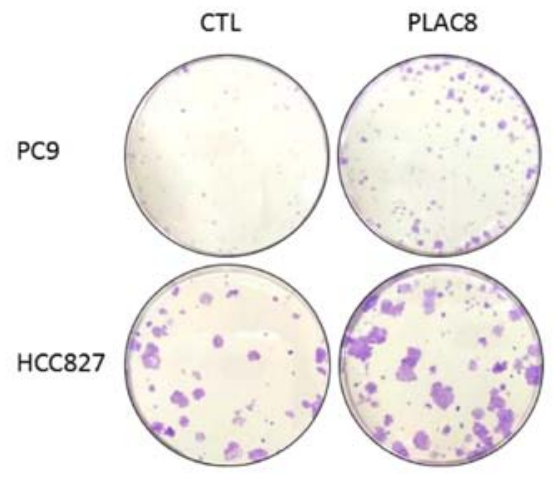

D

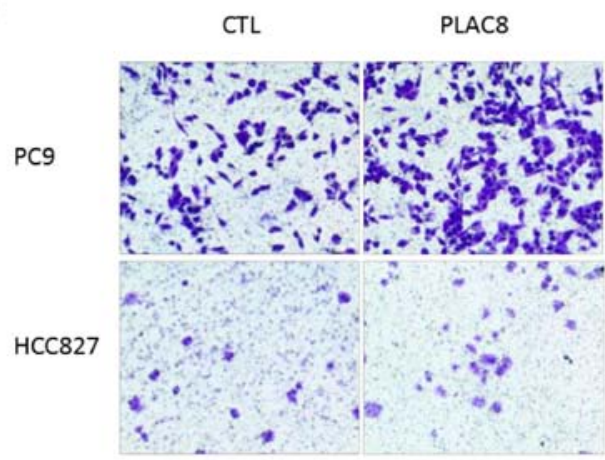

B
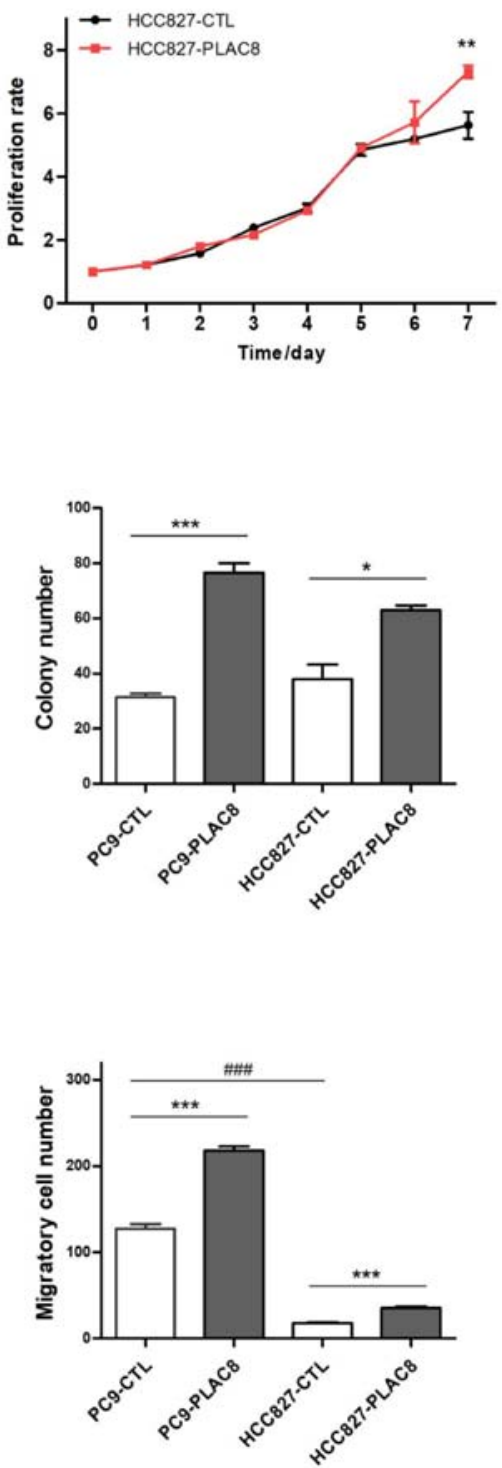

Figure 3. Effect of PLAC8 overexpression on the proliferation, colony formation and migration of non-small cell lung cancer cells. (A) PC9 and (B) HCC827 proliferation ability in control and PLAC8-overexpressing cell lines. Cells were cultured for 7 days and an MTT assay was used to detect the growth ability daily. (C) Colony formation ability of PLAC8-overexpressing cells and the number of colonies following culture for 10-14 days and staining with crystal violet. (D) Migration ability of PLAC8-overexpressing cells and the number of migrated cells following culture for $24 \mathrm{~h}$ in Transwell chambers and staining with crystal violet. ${ }^{*} \mathrm{P}<0.05,{ }^{* *} \mathrm{P}<0.01,{ }^{* * * *} \mathrm{P}<0.001$, and ${ }^{\# \# \#} \mathrm{P}<0.001$. PLAC8, placenta-specific 8; CTL, control.

cells were photographed and counted in at least five randomly selected fields.

Statistical analysis. The data are presented as the mean \pm standard deviation, and an unpaired Student's t-test was used to analyze the statistical significance of the data. Analysis was performed with SPSS software and GraphPad Prism software (version 5; GraphPad Software, Inc., La Jolla, CA, USA). Each experiment was performed at least three times. A statistically significant difference was denoted by a value of $\mathrm{P}<0.05$.

\section{Results}

PLAC8 expression is upregulated in AZD9291-resistant NSCLC cell lines. Increasing evidence has demonstrated that
PLAC8 is associated with various cellular processes, and is an important regulator of tumor progression and resistance (10). In the present study, the PLAC8 levels in AZD9291-resistant and AZD9291-sensitive NSCLC cell lines were initially assessed by RT-qPCR and western blot analyses. As shown in Fig. 1A, the PLAC8 mRNA level in the resistant cells was significantly upregulated, and was almost 100 times higher than that in the parent PC9 and HCC827 cells. To confirm these results, the protein levels of PLAC8 were then evaluated. PLAC8 protein expression was notably upregulated in the resistant cells (Fig. 1B), consistent with the RT-qPCR results. These findings indicated the potential correlation between upregulated PLAC8 levels and AZD9291 resistance in NSCLC.

Overexpressed PLAC8 promotes AZD9291 resistance in NSCLC cells. To investigate whether PLAC8 is involved 

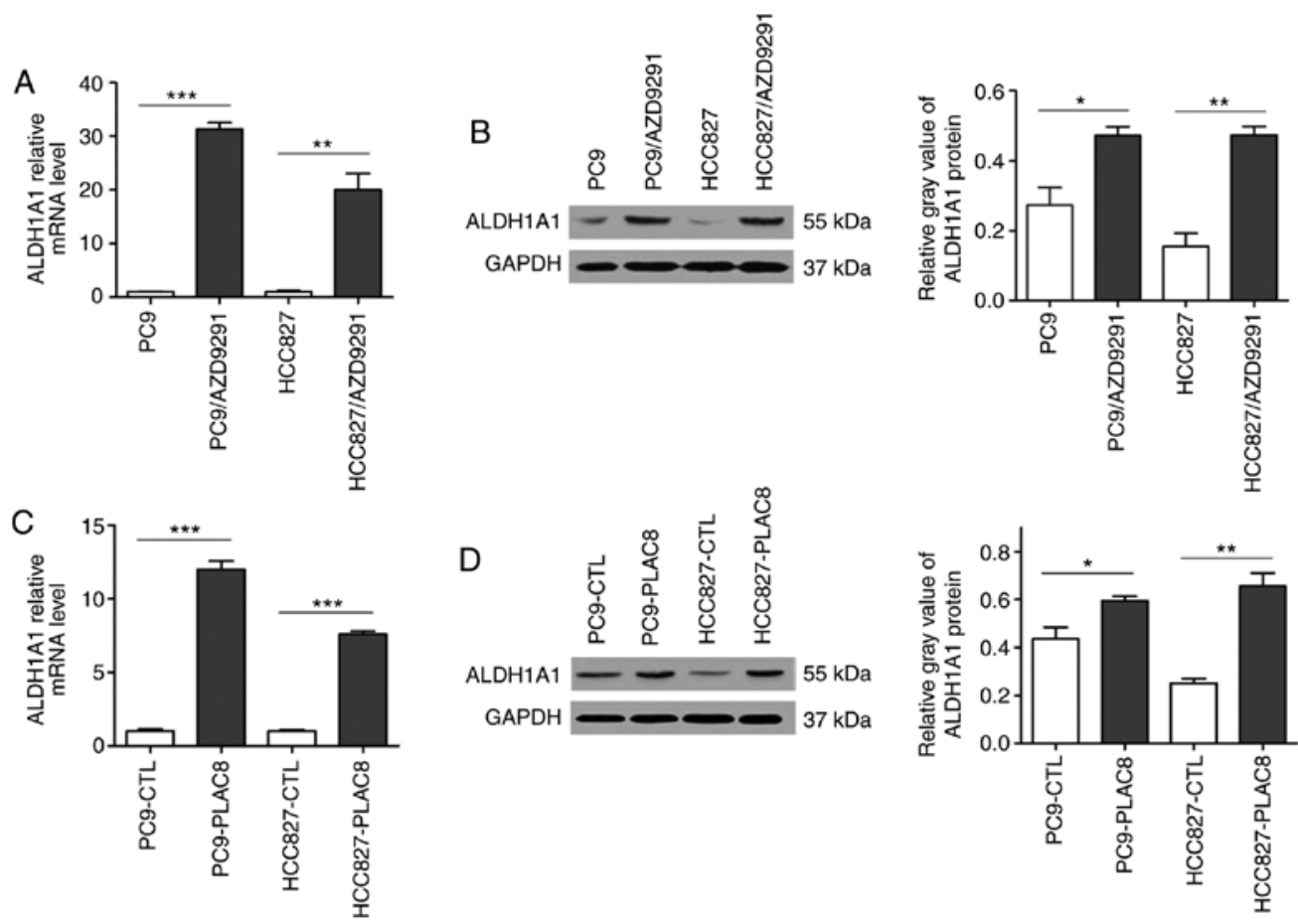

Figure 4. ALDH1A1 levels were upregulated in PLAC8-overexpressing cells. (A) mRNA and (B) protein levels of ALDH1A1 in AZD9291-sensitive and -resistant non-small cell lung cancer cells. (C) mRNA and (D) protein levels of ALDH1A1 in control and PLAC8-overexpressing cells. ${ }^{*} \mathrm{P}<0.05$, ${ }^{* *} \mathrm{P}<0.01$ and ${ }^{* * *} \mathrm{P}<0.001$. ALDH1A1, aldehyde dehydrogenase 1 family member A1; PLAC8, placenta-specific 8; AZD9291, osimertinib; CTL, control.

in AZD9291 resistance in NSCLC, PLAC8 overexpression was induced in PC9 and HCC827 cells (PC9-PLAC8 and HCC827-PLAC8, respectively), and then the $\mathrm{IC}_{50}$ value for AZD9291 was assessed by an MTT assay. As shown in Fig. 2A, the mRNA levels of PLAC8 were significantly upregulated in PC9-PLAC8 and HCC827-PLAC8 cells as compared with those in the control cells (PC9-CTL and HCC827-CTL). The results for PLAC8 protein expression shown in Fig. 2B further confirmed that PLAC8-overexpressing cells were constructed successfully.

The sensitivities of the four cells to AZD9291 were then detected. As shown in Fig. 2C and D, resistance trends were observed in PC9-PLAC8 and HCC827-PLAC8 cells as compared with their corresponding control cells. The $\mathrm{IC}_{50}$ values of the control and PLAC8-overexpressing PC9 cells were 0.163 and $1.019 \mu \mathrm{M}$, respectively, while these values in HCC 827 cells were 0.035 and $0.245 \mu \mathrm{M}$, respectively. These data suggested that the resistance index reached 6-7, thus PLAC8 may serve an important role in AZD9291 resistance in NSCLC (21).

Effects of overexpressed PLAC8 on NSCLC cell proliferation, colony formation and migration. Subsequently, the current study assessed the proliferation, colony formation and migration abilities of PLAC8-overexpressing cell lines. The MTT results indicated that the cell growth patterns of PC9-CTL and PC9-PLAC8 were similar in the first 5 days of culture, whereas PC9-PLAC8 cells exhibited significantly increased growth at 6 and 7 days (Fig. 3A). A similar proliferation trend was observed in Fig. 3B for HCC827 cells. Furthermore, overexpression of PLAC8 significantly elevated the colony formation ability, with a markedly greater number of colonies present in PLAC8-overexpressing cells (Fig. 3C). The migratory cell numbers in the overexpressing cell lines were also significantly increased compared with the corresponding control cells, despite the differential migration ability between the two control cell lines (Fig. 3D).

ALDH1A1 levels are upregulated in PLAC8-overexpressing cells. ALDH1A1 is a putative marker for CSCs in numerous types of tumors and is reportedly overexpressed in certain resistant cells (14). Therefore, the present study examined whether ALDH1A1 is also upregulated in AZD9291-resistant cells. As shown in Fig. 4A and B, ALDH1A1 mRNA and protein levels were significantly increased in resistant cells as compared with those in parent cells, suggesting an underlying link between upregulated ALDH1A1 levels and AZD9291 resistance. Based on the role of PLAC8 in AZD9291 resistance discussed earlier, it was hypothesized that ALDH1A1 expression may be regulated by PLAC8. To confirm this, the levels of ALDH1A1 in PLAC8-overexpressing cells were detected, and it was observed that ALDH1A1 mRNA was significantly upregulated in the overexpressing cells (Fig. 4C). The results of the western blot analysis illustrated a similar protein expression pattern (Fig. 4D).

\section{Discussion}

EGFR-TKIs have demonstrated significant benefits in the treatment of NSCLC with EGFR mutations (22). However, the majority of patients develop resistance within 1 year (23). At present, AZD9291 is the only third generation TKI approved by the FDA. The inevitable acquired resistance to AZD9291 is a significant clinical challenge, and there is an urgent need 
for the identification of possible mechanisms involved in AZD9291 resistance.

Accumulating evidence suggests that PLAC8 is an important player in various cellular processes, thus promoting cancer progression and resistance. Increased expression of PLAC8 facilitates the pro-survival function of autophagy to allow for proliferation in cadmium-transformed prostate epithelial cells, and induces resistance to cadmium toxicity (24). It has been reported that knockdown of PLAC8 expression in clear cell renal cell carcinoma cells significantly reduced the proliferation rates and colony formation ability (13). Additionally, PLAC8 is a critical regulator of autophagy during pancreatic cancer progression (25). In the present study, it was observed that the PLAC8 level was substantially upregulated in AZD9291-resistant cell lines. Overexpression of PLAC8 in sensitive cells contributed to resistance to AZD9291 and significantly increased the cell proliferation, colony formation and migration, which is in agreement with the findings of the aforementioned studies. Notably, increased migration ability indicated potentially higher metastatic ability and tumor malignancy (26). Therefore, PLAC8 may promote increased malignancy in addition to resistance to AZD9291.

CSCs are a unique population of cancer cells identified to have stem-like features, including self-renewal, differentiation and tumorigenesis, which serve an important role in tumor progression and resistance $(27,28)$. A recent study suggested that targeting the surface markers of CSCs may facilitate targeted therapy innovation (29). In the present study, it was demonstrated that ALDH1A1 was upregulated in AZD9291-resistant NSCLC cell lines, consistent with a previous study reporting that overexpression of ALDH1A1 was detected in lung cancer cells that are resistant to afatinib, a second generation TKI (30). Furthermore, cisplatin-resistant A549 cells exhibited elevated levels of ALDH1A1, while knockdown of ALDH1A1 significantly decreased A549/DDP proliferation, increased apoptosis and reduced cisplatin resistance (19). Subsequently, the level of ALDH1A1 in PLAC8-overexpressing cells was assessed in the current study, and the results suggested that the ALDH1A1 level was markedly increased in PC9-PLAC8 and HCC827-PLAC8. These results indicated that the upregulation of ALDH1A1 may be an important mediator between the overexpression of PLAC8 and AZD9291 resistance in NSCLC with increased proliferation, colony formation and migration.

PLAC8 is a relatively small protein, containing a cysteine-rich domain with several CXXC motifs. Jimenez-Preitner et al (7) reported that PLAC8 is required for brown fat differentiation via interaction with $\mathrm{C} / \mathrm{EBP} \beta$, and binds to the $\mathrm{C} / \mathrm{EBP} \beta$ promoter to induce its transcription. Another study by Jimenez-Preitner et al (31) revealed that the transactivating effect of PLAC8 on the C/EBP $\beta$ promoter is critical for white adipocyte differentiation. Therefore, it can be inferred that the elevated ALDH1A1 observed in the present study may be attributed to the transcriptional regulation of the ALDH1A1 promoter by PLAC8, thus inducing resistance. Nevertheless, further studies need to be conducted to investigate the regulatory mechanism of PLAC8 on ALDH1A1 expression.

In conclusion, the current study reported that upregulated PLAC8 prompted NSCLC cell resistance to AZD9291, and significantly elevated the proliferation, colony formation and migration potential. Furthermore, ALDH1A1 may provide a link between the overexpression of PLAC8 and AZD9291 resistance in NSCLC, while PLAC8 may be a potential target for addressing this resistance.

\section{Acknowledgements}

Not applicable.

\section{Funding}

This study was financially supported by the National Natural Science Foundation of China (grant no. 81673014).

\section{Availability of data and materials}

The materials described in this manuscript, including all relevant raw data, will be freely available to any researchers wishing to use them for non-commercial purposes.

\section{Authors' contributions}

GW and XG put forward the conception of the study. XG and XF analyzed and interpreted the data. XF and HS collected and assembled data. All authors contributed to preparation of the manuscript and approved the final version.

\section{Ethics approval and consent to participate}

Not applicable.

\section{Patient consent for publication}

Not applicable.

\section{Competing interests}

The authors declare that they have no competing interests.

\section{References}

1. Heigener DF and Reck M: Lung cancer in 2017: Giant steps and stumbling blocks. Nat Rev Clin Oncol 15: 71-72, 2018.

2. Nagasaka M and Gadgeel SM: Role of chemotherapy and targeted therapy in early-stage non-small cell lung cancer. Expert Rev Anticancer Ther 18: 63-70, 2018.

3. Mayekar MK and Bivona TG: Current landscape of targeted therapy in lung cancer. Clin Pharmacol Ther 102: 757-764, 2017.

4. Saad N, Poudel A, Basnet A and Gajra A: Epidermal growth factor receptor T790M mutation-positive metastatic non-small-cell lung cancer: Focus on osimertinib (AZD9291). Onco Targets Ther 10: 1757-1766, 2017.

5. Jänne PA, Yang JC, Kim DW, Planchard D, Ohe Y, Ramalingam SS, Ahn MJ, Kim SW, Su WC, Horn L, et al: AZD9291 in EGFR inhibitor-resistant non-small-cell lung cancer. N Engl J Med 372: 1689-1699, 2015.

6. Thress KS, Paweletz CP,Felip E, Cho BC, Stetson D, Dougherty B, Lai Z, Markovets A, Vivancos A, Kuang Y, et al: Acquired EGFR C797S mutation mediates resistance to AZD9291 in non-small cell lung cancer harboring EGFR T790M. Nat Med 21: 560-562, 2015.

7. Jimenez-Preitner M, Berney X, Uldry M, Vitali A, Cinti S, Ledford JG and Thorens B: Plac8 is an inducer of C/EBP $\beta$ required for brown fat differentiation, thermoregulation, and control of body weight. Cell Metab 14: 658-670, 2011. 
8. Li Q, Lu TF, Liu D, Hu PF, Sun B, Ma JZ, Wang WJ, Wang KF Zhang WX, Chen J, et al: Screening and analyzing genes associated with Amur tiger placental development. Genet Mol Res 13: 7869-7878, 2014

9. Kaistha BP, Lorenz H, Schmidt H, Sipos B, Pawlak M, Gierke B, Kreider R, Lankat-Buttgereit B, Sauer M, Fiedler L, et al: PLAC8 localizes to the inner plasma membrane of pancreatic cancer cells and regulates cell growth and disease progression through critical cell-cycle regulatory pathways. Cancer Res 76: 96-107, 2016.

10. Uehara H, Takahashi T and Izumi K: Induction of retinol-binding protein 4 and placenta-specific 8 expression in human prostate cancer cells remaining in bone following osteolytic tumor growth inhibition by osteoprotegerin. Int J Oncol 43: 365-374, 2013.

11. Zou L, Chai J, Gao Y, Guan J, Liu Q and Du JJ: Down-regulated PLAC8 promotes hepatocellular carcinoma cell proliferation by enhancing PI3K/Akt/GSK3 $\beta / \mathrm{Wnt} / \beta$-catenin signaling. Biomed Pharmacother 84: 139-146, 2016.

12. Li C, Ma H, Wang Y, Cao Z, Graves-Deal R, Powell AE, Starchenko A, Ayers GD, Washington MK, Kamath V, et al: Excess PLAC8 promotes an unconventional ERK2-dependent EMT in colon cancer. J Clin Invest 124: 2172-2187, 2014.

13. Shi L, Xiao L, Heng B, Mo S, Chen W and Su Z: Overexpression of placenta specific 8 is associated with malignant progression and poor prognosis of clear cell renal cell carcinoma. Int Urol Nephrol 49: 1165-1176, 2017.

14. Xu X, Chai S, Wang P, Zhang C, Yang Y, Yang Y and Wang K: Aldehyde dehydrogenases and cancer stem cells. Cancer Lett 369: 50-57, 2015

15. Koppaka V, Thompson DC, Chen Y, Ellermann M, Nicolaou KC Juvonen RO, Petersen D, Deitrich RA, Hurley TD and Vasiliou V: Aldehyde dehydrogenase inhibitors: a comprehensive review of the pharmacology, mechanism of action, substrate specificity, and clinical application. Pharmacol Rev 64: 520-539, 2012.

16. Yu J, Alharbi A, Shan H, Hao Y, Snetsinger B, Rauh MJ and Yang X: TAZ induces lung cancer stem cell properties and tumorigenesis by up-regulating ALDH1A1. Oncotarget 8 : 38426-38443, 2017.

17. Duong HQ, You KS, Oh S, Kwak SJ and Seong YS: Silencing of NRF2 reduces the expression of ALDH1A1 and ALDH3A1 and sensitizes to 5-FU in pancreatic cancer cells. Antioxidants (Basel) 6: pii: E52, 2017.

18. Alamgeer M, Ganju V, Szczepny A, Russell PA, Prodanovic, Z, Kumar B, Wainer Z, Brown T, Schneider-Kolsky M, Conron M, et al: The prognostic significance of aldehyde dehydrogenase 1A1 (ALDH1A1) and CD133 expression in early stage non-small cell lung cancer. Thorax 68: 1095-1104, 2013.

19. Wei Y, Wu S, Xu W, Liang Y, Li Y, Zhao W and Wu J: Depleted aldehyde dehydrogenase $1 \mathrm{~A} 1$ (ALDH1A1) reverses cisplatin resistance of human lung adenocarcinoma cell A549/DDP. Thorac Cancer 8: 26-32, 2017.
20. Livak KJ and Schmittgen TD: Analysis of relative gene expression data using real-time quantitative PCR and the 2(-Delta Delta C(T)) method. Methods 25: 402-408, 2001.

21. Lai YH, Lin SY, Wu YS, Chen HW and Chen JJW: AC-93253 iodide, a novel Src inhibitor, suppresses NSCLC progression by modulating multiple Src-related signaling pathways. J Hematol Oncol 10: 172, 2017.

22. Tan CS, Gilligan D and Pacey S: Treatment approaches for EGFR-inhibitor-resistant patients with non-small-cell lung cancer. Lancet Oncol 16: e447-e459, 2015.

23. Yu HA, Arcila ME, Rekhtman N, Sima CS, Zakowski MF, Pao W, Kris MG, Miller VA, Ladanyi M and Riely GJ: Analysis of tumor specimens at the time of acquired resistance to EGFR-TKI therapy in 155 patients with EGFR-mutant lung cancers. Clin Cancer Res 19: 2240-2247, 2013

24. Kolluru V, Pal D, Papu John AMS, Ankem MK, Freedman JH and Damodaran C: Induction of Plac8 promotes pro-survival function of autophagy in cadmium-induced prostate carcinogenesis. Cancer Lett 408: 121-129, 2017.

25. Kinsey C, Balakrishnan V, O'Dell MR, Huang JL, Newman L, Whitney-Miller CL, Hezel AF and Land H: Plac8 links oncogenic mutations to regulation of autophagy and is critical to pancreatic cancer progression. Cell Rep 7: 1143-1155, 2014.

26. Hanahan D and Weinberg RA: Hallmarks of cancer: The next generation. Cell 144: 646-674, 2011

27. Leon G, MacDonagh L, Finn SP, Cuffe S and Barr MP: Cancer stem cells in drug resistant lung cancer: Targeting cell surface markers and signaling pathways. Pharmacol Ther 158: 71-90, 2016.

28. Prieto-Vila M, Takahashi RU, Usuba W, Kohama I and Ochiya T: Drug resistance driven by cancer stem cells and their niche. Int J Mol Sci 18: pii: E2574, 2017.

29. MacDonagh L, Gray SG, Breen E, Cuffe S, Finn SP, O'Byrne KJ and Barr MP: Lung cancer stem cells: The root of resistance. Cancer Lett 372: 147-156, 2016.

30. Hashida S, Yamamoto H, Shien K, Miyoshi Y, Ohtsuka T, Suzawa K, Watanabe M, Maki Y, Soh J, Asano H, et al: Acquisition of cancer stem cell-like properties in non-small cell lung cancer with acquired resistance to afatinib. Cancer Sci 106: $1377-1384,2015$

31. Jimenez-Preitner M, Berney X and Thorens B: Plac8 is required for white adipocyte differentiation in vitro and cell number control in vivo. PLoS One 7: e48767, 2012.

This work is licensed under a Creative Commons Attribution-NonCommercial-NoDerivatives 4.0 International (CC BY-NC-ND 4.0) License. 